\title{
Ageing and China: Towards Theory, Policy and Practice
}

\author{
Jason L. Powell* \\ Department of Social and Political Science, University of Chester, \\ $\mathrm{CH} 1$ 4BJ, United Kingdom \\ *E-mail address: Jasonpwll3@gmail.com
}

\begin{abstract}
In the $21^{\text {st }}$ Century, economists and social analysts around the about the rising numbers of older people in their society. Ther inadequacy of pension funds, of growing pressures on welfa shrinking numbers of younger people to carry the burden of tors. article focuses on such gerontological issues in China, where the older people hay become a rapidly panding proportion of the population. While resources do need to be targeted on he vulnerable older people, the presumption that older people as a whole are an economic and social urden must $b$ questioned. This is an ageist view that needs to be combated by locating how bio-med views on ging seep into policy spaces in China that position negative perceptions of ag as both and populational problems. The article then moves to observe the implications of older people in China in terms of "vulnerable" aging but deconstruct such "fixed ex ian is by juxtaposing active aging as key narrative that epitomizes "declining to decline" as oused oy bio-medical sciences.
\end{abstract}

\section{INTRODUCTIC}

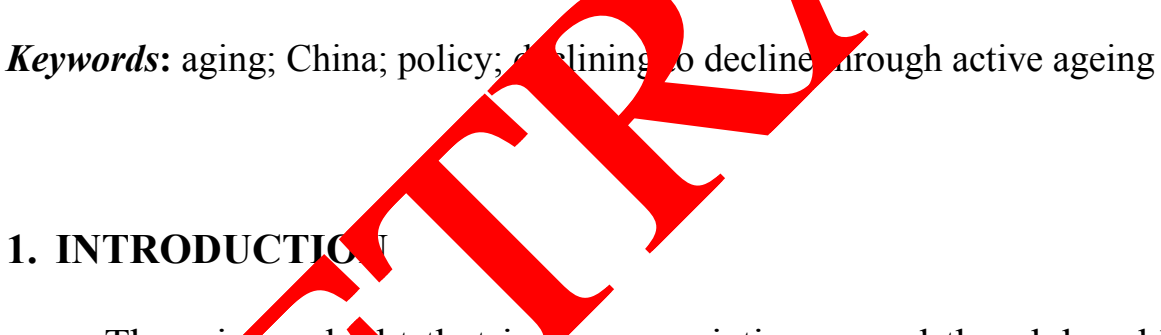

There $\mathrm{j}$ no do $\mathrm{t}$ that in many societies around the globe older people are a growing proportion or obal p pulation (Krug 2002). There are 7.1 billion people living on the planet earth in 2 youn ser p ple pre nated to a society in which people in later life constitute a substantial pr. tion of the to al population. While the biological and psychological models of aging inscr "inevitable" and "universal" process, an aging population is neither (Phillips 1998). Transformations in the age profile of a population are a response to political at economic structures. A major concern for organizations such as the United Nations and World Bank focuses on the number of such "dependent" older people in world society (Krug 2002).

Indeed, older people in particular constitute a large section of populations in global aging. In relation to public services that have to be paid for by "younger" working people, the percentage of the population has been used to signify such "burdensome" numbers. Not only are older people seen as dependent but also children under school leaving age and people over the retirement age. Dependency rates - that is, the number of dependants related to those of working age - altered little over the twentieth century and yet the notion of "burden" group 
retains its legitimacy. The reason there has been so little change during a period of so-called rapid aging populations is that there has been a fall in the total fertility rate (the average number of children that would be born to each woman if the current age-specific birth rates persisted throughout her child-bearing life) (Phillipson, 1998).

Changes in the age structures of all societies also affect total levels of labor force participation in society, because the likelihood that an individual will be in the labor force varies systematically by age. Concurrently, global population aging is projected to lead to lower proportions of the population in the labor force in highly industrialized nations, threatening both productivity and the ability to support an aging population (Powell 2009). Coupled with rapid growth in the young adult population in Third World countrie o, the $\mathrm{d}$ Bank (1994) foresees growing "threats" to international stability, p "g differ demographic-economic regions against one another. That the United Nation $(20$ views $t$ relationship between aging populations and labor force participation $\mathrm{y}$ th panic gni es important policy challenges, including the need to reverse recent ty ds to $\mathrm{dd}$ dee asing labor force participation of workers in late-middle and old age de vito datory trement in both Western and Eastern countries such as the UK (Jackson and Pow 2001) and China (Chen and Powell, 2011).

Notwithstanding this, in China there is also an gn ing increase in the aging population that replicates global trends. It can be s on the aging population has increased from just over 4.4 percent t just under 7 percent from 1953 to 2000 (Cook and Murray 2001). Note that the increase has ot been con tant, reflecting the negative impact of the Great Leap Forward and the successiv amines u on the demographic profile, shown in the results of the 1964 Census.

Since that latter date, the percentage o derly has nearly doubled, while the actual numbers have more than tripled, being approx maty times that of 1964 by the year 2000 . By 2000, there were 88 million Chinace aged o or over, compared to just under 25 million in 1964, an increase of 63 million ras ih

As is well known, C 6 years.

lation policy, most usually referred to as the Single Child Family Program ou un anese commentators regard the phrase as a misnomer, given that $\mathrm{m}$ than one $1 \mathrm{~d}$ is possible within a number of situations), has led to a rapid deceleration $\mathrm{i}$ the th rate, $\mathrm{w}$, ch was only 13.38 per thousand in 2001 , compared to 21.06 in 1990 and 8.5 in 1 (Cook and Murray 2001).

This con oversial polic, ambasted and praised in equal measure depending on perspective, mea rapid expansion in the number of single children only households at the very same that proportion of the elderly has also increased as a new era of prospen re. d man households in China.

Estin tes sugg $r$ that up to 300 million less people have been born as a result of this prou of and ot to suggest that improved living standards via modernization would have led to the same out voluntarily as growing numbers of urban dwellers in particular chose to reduce their famil/s size. This last point hints at the important spatial dimension of demographic change in China. There is on the one hand a marked contrast between urban and rural life in China and, closely related to this, a marked contrast between Eastern China and Western China (Cook and Powell, 2007). It is in the heavily urbanized "Gold Coast" of the Eastern Seaboard in which China's spatial transformation is most dramatic, with fast-expanding cities being especially concentrated in the Pearl River delta of the Southeast and the Yangtse Delta in Central East China (Cook and Murray 2001). 
Hence, it is not simply that the Chinese government has belatedly recognized "the greying" of populational constructions and policy implications, it is that they continue to look for knowledge of aging as the power to define old age as a social problem in terms of dualistic distinctions between deviancy and normality. An aging population, like that of an individual being studied by bio-medical models, is seen as a "burden" problem in terms of economic management of Eastern (and Western) economies.

It could be argued when looking at the effects of a so-called "demographic time bomb" across US, Europe, and Asia, it may have been grossly exaggerated.

Such old age, therefore, has been perceived negatively via a process of "aoriam"stereotyping older people simply because of their chronological age. Agist stereo $s$ pes Su "aging populations" act to stigmatize and consequently marginalize old people a differentiate them from groups across the life-course who are not labeled "old Qythewa 1993).

One of the ways to interpret social aging such as being a catego, ation hether oe in individual or populational terms is through use of theorizing or wh means age in society; that is, concerns and social issues associated with agin and the in wich these themes are influenced and at the same time influence the so io which po live. Thus, to understand the process of aging, looking through the lens f the ciological imagination" is not to see it as an individualized problem rather as a peral issue t.
World and Third World nations as a whole.

In supporting this latter view, there is a need $t$ focus on ho populational discourses of aging in China are influenced and reinforced by bio edical models of aging that help drive perceptions of older people as a burdensom aroup.

\section{WHERE DOES THE AMBIVAIENCE S AGKG COME FROM?}

There are important imp tion for how ging is viewed by not only in terms of global

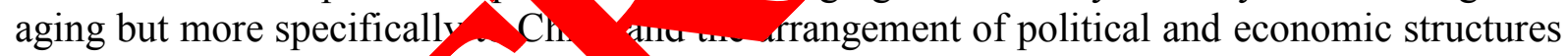
that create and sanctic social po es grounded in knowledge bases of "burdensome" populations (cf Powe', 24 Such ki, wledge bases are focused on: one, "biological aging" which refers to ha inter and external physiological changes that take place in the individual body, twa, psychor cal aging is understood as the developmental changes in mental funct ing - otional and cognitive capacities.

Bio-med theories of aging can be distinguished from social construction of aging: (1) foc on bio-sychological constituent of aging, and (2) on how aging has been soci dly ce structed. ne perspective is driven from "within" and privileges the expression fron ne mor werlds. The other is much more concerned with the power of external structu that shape individuality. In essence, this social constructionism poses the problem from the sective of an observer looking in, whilst the biomedical model takes the stance of inside the individual looking out (Powell and Biggs, 2000). There has long been a tendency in matters of aging and old age to reduce the social experience of aging to its biological dimension from which are derived a set of normative "stages" which over-determine the experience of aging.

Accordingly being "old," for example, would primarily be an individualized experience of adaptation to inevitable physical and mental decline and of preparation for death. The paradox of course is that the homogenizing of the experience of old age which the reliance on 
the biological dimension of old age entails is in fact one of the key elements of the dominant discourse on aging and old age.

It is interesting that comparative historical research on aging in Eastern culture highlights an alternative perception of aging; in $18^{\text {th }}$ Century China has highlighted a rather different path as to the conceptualization of aging as a scientific process developed by western rationality. For example, Cook and Powell (2003) observe that traditional Chinese society placed older people on a pedestal. They were valued for their accumulated knowledge, their position within the extended family, and the sense of history and identity that they helped the family to develop (Murray,1998). Respect for elderly people was an integral nart of Confucian doctrine, especially for the family patriarch.

This was a view that was also prevalent in Ancient Greece with the notio

"respect" for older people especially regarding gendered issues of p archy Bytheway, 1993). Prior to industrialization, in India, there was a bo sowment of er people had responsible leadership roles and powerful decision-maki posit ns hed se of their vast "experience," "wisdom" and "knowledge" (Katz, 1996).

It seems with the instigation of Western science and $r$ lonality, ing $b$ dgan to be viewed in a different more problematic context than to th nfucian a rine of aging epitomized in China and issues of respect for aging in Indi. Mar. Yeidegger (1971) makes the similar point when he spoke of the Westernization
Western science and language.

Indeed, the technological developments due industriali ation, Westernization, and urbanization-under the purview of distorted fon of mode nity-have neglected these statuses of aging by downgrading its concentualization understanding individuality in Western culture, the birth of "science" gav mate credibility to a range of bio-medical disciplines of whom were part of its umb lla lit ticular, the bio-medical model has become one of the most controvercial yet $p$ verful of both disciplines and practice with regard to aging (Powell and Bigo,, 20 ).

The bio-medical mode 1 rese ts the contested terrain of decisions reflecting both normative claims and tech og wossondes. Bio-medicine refers to medical techniques that privilege a biologis and psyc gical understanding of the human condition and rely upon "scientific assu ptr " that pos on attitudes to aging in society for their existence and practice. Hence, tio tific, ticine is based on the biological and psychological sciences. Some doctrines of the biomedy model more closely reflect the basic sciences while others refer to the nary cern of medicine, namely diseases located in the human body. Most important is th. ese bel fs hold together, thereby reinforcing one another and forming a cohere ientat tow d the mind and body. Indeed, the mind-body dualism had become the ocatio of regin $a$ and control for emergence of scientific in a positivist methodological seal for 4. model.

In t. anse, bio-medicine is based on the biological and psychological sciences. Some doctrines of the biomedical model more closely reflect the basic sciences while others refer to the primary concern of medicine, namely diseases located in the human body. Most important is that these beliefs hold together, thereby reinforcing one another and forming a coherent whole (Powell, 2001). By developping an all-encompassing range of bio-medical discourses, many forms of social injustice could be justified as "natural," inevitable and necessary for the successful equilibrium of the social whole such as mandatory retirement and allocation of pensions (Phillipson, 1998). 
Bio-medical gerontology is a fundamental domain where medical discourses on aging have become located and this is very powerful in articulating "truths" about aging. Under the guise of science and its perceived tenets of value-freedom, objectivity and precision (Biggs, 1993), bio-medical gerontology has a cloth of legitimacy. Biological and psychological characteristics associated with aging have been used to construct scientific representations of aging in modern society. The characteristics of biological aging as associated with loss of skin elasticity, wrinkled skin, hair loss or physical frailty perpetuates powerful assumptions that help facilitate attitudes and perceptions of aging. It may be argued that rather than provide a scientific explanation of aging, such an approach homogenizes the experiences of aring by suggesting these characteristics are universal, natural and inevitable. These ass inption powerful in creating a knowledge base for health and social welfare professio $\mathrm{l}_{\mathrm{s}}$ who wo with older people in particular medical settings such as a hospital or genem do ss surge and also for social workers (Powell 2009).

These new forms of social regulation were also reflected the mily a the community. Hence, modern systems of social regulation have bo on reasing blurred and wide-ranging (Powell, 2009). Increasingly, modern socie \& regula the populational construct by sanctioning the knowledge and practices a new ho sciencesparticularly psychology and biology. These are called gero tolog "epistemes" which are "the total set of relations that unite at a given period, th a cursive $p$ tices that give rise to epistemological figures, sciences and possibly forn alized systems" (roucault, 1972: 191). The "psy" complex or bio-medical epistemes refers the networl of ideas about the "nature" of individuals, their perfectability, the reasons for ir behavis and the way they may be classified, selected and controlled. It aip to man improve individuals by the manipulation of their qualities and attribute dependent upon scientific knowledge and professional interventions and expertise. I Im 1 ities are seen as measurable and calculable and thereby can be chanoed and in roved? The new human sciences had as their central aim the prediction of futy e bo vior (Po vell, 2009).

Powell and Biggs (2009 oges that a prevailing ideology of ageism manifests itself in the bio-medical model via sug. Iun persons with such biological traits have entered a spiral of decay, declir and deter tion. Along with this goes certain assumptions about the ways in which $\mathfrak{p}$ op ith outwa signs of aging are likely to think and behave. For example, there aress.dmpti that "older people are poor drivers" or that older people have little interest iv relationships / involve sexual pleasure that are all explained away by "decline" an "deter" thion" master narratives that comprise an aging culture. The effects of the "decline" a decay" nalogies can be most clearly seen in the dominance of medicotechnic lution th problems that aging and even an "aging population" (Phillipson, $199^{\circ}$ is th aght to 3 . Here, the bio-medical model has both come to colonize notions of age re re come to stand for the proces aging itself (Powell, 2001).

Est Md Binney (1989 cited in Powell, 2001) have used the expression "biomedicanation of aging" which has two closely related narratives: (1) the social construction of aging as a medical problem, and (2) ageist practices and policies growing out of thinking of aging as a medical problem. They suggest:

"Equating old age with illness has encouraged society to think about aging as pathological or abnormal. The undesirability of conditions labeled as sickness or illness transfer to those who have these conditions, shaping the attitudes of the persons themselves and those of others towards them. Sick role 
expectations may result in such behaviors as social withdrawal, reduction in activity, increased dependency and the loss of effectiveness and personal control-all of which may result in the social control of the elderly through medical definition, management and treatment" (Estes and Binney, 1989, quoted in Powell, 2001: 119).

These authors highlight how individual lives and physical and mental capacities that were thought to be determined solely by biological and psychological factors, are, in fact, heavily influenced by social environments in which people live. This remains invisiblo to the bio-medical approach because they stem from the societal interaction befor beco $g$ embedded and recognizable as an "illness" in the aging body of the person. F example, the "sociology of emotions" the excursion of inquiry has proposed that "rress not on rooted in individualistic emotional responses but also regulated, class ied and ved oy social norms of western culture (Powell and Biggs, 2000).

This type of research enables the scope of aging to be bro wen byond b medical individualistic accounts of the body. On this basis alone, sociolo $y$ invites to re ognize that aging is not only a socially constructed problem by bic th ical scien but also the symptomatic deep manifestation of underlying relations o pow nd inequality that cuts across and through age, class, gender, disability and sox ality (P 11 and Biggs, 2000;
Powell, 2001).

At this level of analysis, sociology addresse bio-medicin as one of the elements of social control and domination legitimated through p r/knowle ge of "experts" (Powell and Biggs, 2000). Such expert formation has als been labo oist (Bytheway, 1993). Ageism is where the assumptions made about old a individuals but as a homogenous group, w ich discriminated against (Bytheway, 1993).

Chinese society uses age cato ries to divide this ongoing process into stages or segments of life. These life st are cocially constructed rather than inevitable. Aging, too, is a production of social cr ory any puit of life span, age simultaneously denotes a set of social constructs, de ed by the rms specific to a given society at a specific point in history. Thus, a spe fic iod of lit infancy, childhood, adolescence, adulthood, middle age or old age is frenced the structural entities of a given society. Therefore, aging is not to be considered the $\mathrm{m} /$ product of biological-psychological function rather a consequence $f$ soc ultural hactors and subsequent life-chances. Indeed, society has a number of cul $y$ and s cially defined notions of what Phillipson (1998) calls the "stages of life" vever, wnd mental question is how bio-medical gerontology has stabilized itself witl a pos vist dis arse that not only reflects history but also the total preoccupation with the by of ang that have important implications for older people and health lifestyles in Chis

\section{BIO-MEDICINE, FAMILY CARE, AND AGING: IMPLICATIONS FOR CHINA}

The dominant bio-medical discourse of aging in China dwells on the processes of physical deterioration associated with becoming older. In this perspective, the aging body has to deal with increased levels of incapacity, both physical and mental, and becomes increasingly dependent on younger others for sustenance and survival; it is the family through informal care that has to provide care of older people who may have illnesses, according to 
the Law on the Rights and Interests of the Elderly, introduced by the PRC in 1996. The biomedical problematization of aging has secreted wider questions of power and inequality; especially influential is occidental modernity. A powerful discourse is thus developed which follows that of the West, via notions of "social inclusion" and "family care," and the allimportant role of the consumer in buying products for the elderly, from disability aids through to private pensions (Powell and Cook, 2001). The latter suggest that this process constructs the aging body as a site of surveillance by the Chinese state, constructing them as, following Foucault, objects of power and knowledge in which "its your age" is the prevailing authority response to the elderly "customer."

Powell and Cook (2001) have further noted that older people will be increas y probed for social, psychological and economic factors such as "frailty" or "er cted level supervision." "There are indications, for example, that where care homes ree pr red, the are for the more active elderly, rather than those in greatest need" (Powe' and Cook, $01 \%$ ).

This Foucauldian point has been borne out via an article on angh China Jaily, 2004) with "most nursing homes in Shanghai have entry criteria th ta arro minority of elderly people. Some admit only those who are capable of in ependen ing nile others accept only bedridden patients. While dementia is a comm ndition an the elderly, those afflicted by it are generally excluded by the criteria" yen in seriously, if a patient's condition changes according to these criteria, he or she wo to to home. "The lack of a continuum of care creates devastating situati is for the patien s and their families." Further, nursing homes have only minimal level of 1 dical suppo t available, and patients are transferred to hospitals too readily if they have an nent muc beyond the common cold. The patient can then lose their place in the pursing hon bed is transferred and thus be subject to further stress. "Preventive care, therapy, and spiritual care, which are crucial components of care for the elderly, an ger crom overlooked. Many nursing homes do not provide such services out of conearns for c st or accident liabilities." The Shanghai article also notes, damningly, that:

"The financial ro erm care accumulates on an elderly population alread anduring ter, budget constraints because of retirement and unemploy ent the abse ce of government subsidy, the higher fees charged by nustain nursing homes deprive elderly people with limited financial neans of their a ss to care" (China Daily, 2004).

In the lis these other issues the PRC government is attempting to change the ways jo ich th "derl are perceived, via campaign slogans such as "respect the elderly" and peop first." former campaign seeks to encourage younger people to visit the efac on sense of isolation that the elderly can feel, to look on or their needs out on the streets and to generally raise awareness of the situation of older peo ${ }_{1}$ There is a resonance here with ancient Confucian tradition in which the elderly were venerated. There have also been attempts to encourage younger people to think about "healthy aging," but this is meant in terms of ensuring that they themselves have adequate financial provision as they age. "People first" is the attempt to recognize that "aging is an individual-specific process" and that "a functional healthcare system for the elderly should integrate all aspects of care ... emphasizing and fulfilling individual needs and preferences" (CD, 2004).

Older people in rural areas are more likely to have to face emigration of their children to the cities as China's urbanization proceeds apace. This can leave them physically and socially 
isolated in a remote rural area, no longer able to rely upon their family to look after them in their old age, as was once the tradition.

Indeed, as social norms and values change, younger people may no longer be willing, even when they are able, to support elderly parents, and in recent years the law has been used to take children to court in order to force them to support their parents. For example, a new law came into force on October 1, 1996 on "The Rights and Interests of the Elderly" that explicitly states that: "the elderly shall be provided for mainly by their families, and their family members shall care for and look after them" (Du and Tu, 2000).

Notwithstanding the legal process, at its most extreme, the concerns of elderlw neople are expressed via suicide-gerontocide. The elderly can struggle in the face of me ma e social changes that China is facing, and the abandonment of the tradition which th themselves would have looked after their own parents and grandparents. They $y$ feel stressed and alienated that suicide seems the preferred option.

For example, a 76 year old man blew himself up in a courtroom protedurin $/$ case against his family, who had offered only 350 Yuan a month to sy por 1 when 00 Yuan was required (8 Yuan $=1$ USD) (Cook and Murray 2001). In ociet, of rapid transformation, older people in particular may be vulnerab cons sendonment within a more materialistic and selfish new world eni nized the forces of global capitalism and seeping impingement into day to day liy older po in China.

\section{DECLINING TO DECLINE-ACTIVE AGIN}

At the other end of the scale is the act aldy, probably the ideal state for all elderly people. Briefly, older people traditionally we mo to be active within rural areas of China, in part because they had to he in ord to mantain their livelihood. This particular tradition continues today; officiz' dato or the 10 s showed that 26 percent of people in rural areas still depended on their $Q$ abo earnings ompared to only 7 percent in urban areas.

This is not about ady tin a people should have to work to continue to earn a living, but we are su esting tha active lifestyle be promoted where possible. In the cities of China toda it eartening $\sigma$ see the colonization of open space throughout the hours of daylight a aven the evening by the elderly who are engaged in a wide variety of activities, from the traditio /(such as taijiquan and qigong), walking one's pet bird, traditional $d$ ce or petry writing using brushes dipped in water only, illustrating the ephemeral and sing nat e of life itself). Hopefully, the increased pollution which China's cities f vill no rode he potential gains from these and other activities for older people. An altern ve diso rse on aging can point to ways in which the elderly can be dep, ven a negative medical, economic, political and social category.

must begin with appreciation of older people first of all as people rather than as a category all 2006). Older people share, however, apart from their longevity, a wide and deep experence of life itself, and thus of life situations. In China, older people used to be venerated because they were, almost literally, the founts of wisdom, the holders of accumulated knowledge far in advance of the younger members of the family and community. Today, knowledge is far less likely to be oral, and far less dependent on accumulation by the individual. Instead, it is increasingly available at the touch of a button via an Internet search engine, even if there are some restrictions on web provision in the PRC. But, it can be suggested, there should still be a major role for the accumulated wisdom of older people's experiences as carriers of historical wisdom. 
In China, there is a growing awareness of the need to have a sophisticated, multidimensional policy to respond to the needs of older people. But there is still a strong biomedical emphasis on surveillance and control. It could be suggested that policy needs to be driven by the elderly themselves wherever possible. They should be encouraged to define and state their own needs, provided with support when this is required, but the overriding emphasis should be on providing support that fosters active lifestyle and independent living wherever and whenever possible. This means encouragement to the elderly to share their accumulated experience, to provide their oral histories and their views on the momentous changes that they, along with China itself, have lived through. Older people should be-rolued, and involved in wider society on terms that they themselves desire, recognizing that a $h$ for privacy and seclusion might be their preference.

\section{CONCLUSION}

Finally, it is worth noting that cases exist where narratiy $s$ and mis histo les coexist and play a role in producing and strengthening social excly ol The med "gaze" refers here to discourses, languages, and ways of seeing that shas the vistanding of aging into questions that center on, and increase the power of, ate, and or de-legitimize other possibilities. A consequence is that areas of $\mathrm{p}$ licy may at first) seem tangential to the medical project come to be reflected in its pa icular disto ting mirror. The Chinese "Duomin"-a subcategory of a wider population "ficially clalogued as fallen people, beggars or ruined households - were seen a inferior demned to bear low status on account of a number of beliefs prevailing a a creational myth that asserts that the Do mi closely related to Chinese ethnic minorities like the She and Yao and that al these groups shared the belief in Pan-hu, a common dog ancestor. As to mi $\mathrm{O}-\mathrm{h}$,ries, $\mathrm{w}$ run into different stories which state that the Doumin were either: (1) des lants of Song Dynasty traitors, deserters or prisoners; (2)

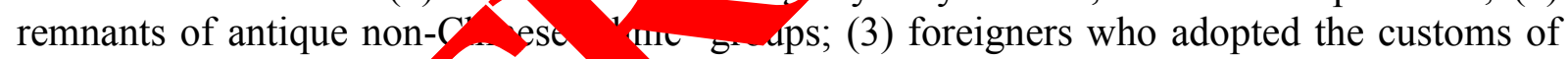
Chinese lower social $s$ ta; and $(4$ escendants of domestic slaves. Yet in all cases the Doumins excluded a d o ste statu came about as a punishment society bestowed upon them. They were anced th erforming polluting occupations (ox head lanterns making, ironwork, barb s, caretakers, h -catching, entertaining, among others) and limited to live in segregated o ters $c$ vide town. Furthermore, the Doumin were not allowed to study or take public office, serve a officers and were obliged to marry among themselves. This sugges then cial dentity was a result of the legal status imposed on them and not the othe way ound. A Aansson (1996: 87) expresses it: "Once fallen people had been labeled as ar ar behom little choice but to conform to the behavior expected from people who had tho cial 1dentity associated with their legal status." For older people if they are regarded as an infe. eategory then their behavior will begin to mimic this categorization. "Dependent is as deperdent does" is a major danger in the continuation of the dominance of the biomedical model of aging.

A key point here is that the notion of the "bio-medical gaze," not only draws our attention to the ways that aging has become "medicalized" as a social issue in China, it also highlights the way in which older people are encouraged for as long as possible to "work on themselves" as active subjects. Thus, as Blaikie (1999) has pointed out, older citizens are encouraged to take greater personal responsibility for their health and for extending this period of their active aging. Those who are defined in relation to their health then discover 
themselves transformed into passive objects of medical power in China. How do we go beyond this in managing old age?

We could suggest macro-social practices have become translated into particular ways of growing old that not only shape what it is to age successfully, but are also adopted by older adults, modified to fit their own life circumstances and then fed back into wider narratives of aging well. Harry R. Moody (1998) coins this as the "Illderly" and "Wellderly" and managing aging experiences is about resistance to dominant discourses of bio-medicine. According to Frank (1998), the personal experience of illness is mediated by bio-medical procedures that shape and contribute to how the older people recognizes their own process of ill heolth and recover. Katz (2000) notes that the maxim of "activity for its own sake" as a mean ff managing later life not only reflects wider social values concerning work ar non-work, also provides personal means of control and acts as grounds for resistance. additic Phillipson (1998) argues that changes in westernized policies has occy red from ing old age as a burden to seeing it as an opportunity to promote productive ging. bis reh is an attempt to shape acceptable forms of aging whilst encouraging $g$ en $1 \mathrm{~s}$ to se monitor their own success at conforming to the challenging paradigm hegemo of bo-medicine and its neglect of the human agency of older people.

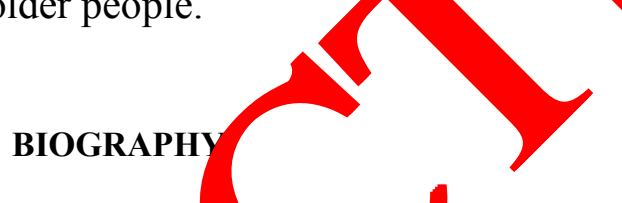

Hon Professor Jason L. Powell BA (Hons), MA, Ph.D, SA is Unive ity Lecturer, Honorary Professor and Adjunct Professor. He is formerly Professor of Social Gero oy and ssociate Dean of Faculty of Health and Life Sciences at University of Coventry. He Howship at University of Liverpool; Honorary Professor at Australia-Asia Research and wan Foundation at Tasmania University; Visiting Research Fellow at Oxford; and recently invited as Vi ting Aarvard University. He has been Visiting Professor in Canada, US, Australia, Africa and Jorda Ae was nominated and elected to Fellowship of the British Royal Society of Arts (FRSA) nition o his research. He has strong interests in social theory, ageing, power and identity. He is autb rof So al Theory Ageing (2006) which was part of Charles Lemerts distinguished „New Social Formation ook risc Row nan and Littlefield: New York.

\section{References}

[1] China D ly (20 4), "Tailo, /ng Health Care Policies for the Elderly", April 15 2004.

[2] Chen, S a owell, J (Eds.) Aging in Perspective and the Case of China, Nova

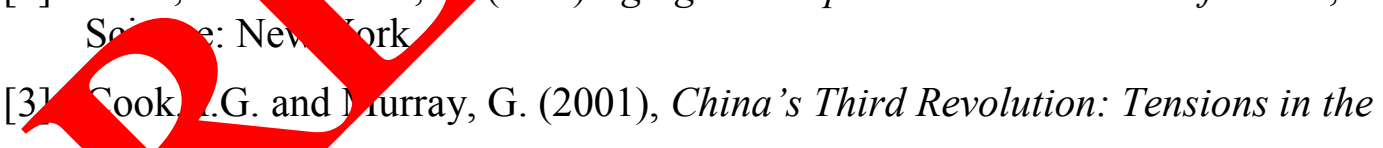

[4] 1. Fition to Post-Communism, Curzon: London.

[5] Du, P Ad Tu, P. (2000), Population Ageing and Old-Age Security, in W.Z. Peng and Z.G. Guo (eds.), The Changing Population of China, Blackwell: Oxford, UK.

[6] Foucault, M. (1972), The Archeology of Knowledge, Tavistock: London.

[7] Frank, A.W. (1998), Stories of Illness as Care of the Self: A Foucauldian Dialogue, Health, Vol. 2, No. 3, pp. 329-48.

[8] Government of China (2002), China Statistical Yearbook 2002, China Statistics Press: Beijing. 
[9] Hadley, R. and Clough, R. (1996), Care in Chaos, Continuum International: New York.

[10] Hansson, A. (1996), Chinese Outcasts: Discrimination and Emancipation in Late Imperial China, E.J. Brill: Leiden, The Netherlands.

[11] Harper, S. (1994), China's Population: Prospects and Policies, in D. Dwyer (ed.), China: The Next Decades, Longman Scientific and Technical: Harlow, UK.

[12] Heidegger, M. (1971), Poetry, Language, Thought, Harper and Row: New York.

[13] Katz, S. (1996), Disciplining Old Age: The Formation of Gerontological Knowledoe, The University Press of Virginia: Charlottesville, VA.

[14] Katz, S. (2000), Busy Bodies: Activity, Aging and the Management of E day Life, Journal of Aging Studies, Vol. 14, No. 2, pp. 135-52.

[15] Krug, E.G. (2002), World Report on Violence and Health, World cealth Prganı. Geneva, Switzerland.

[16] Liu, J. and Lin, F. (2000), Long-Term Effect of China's Far 1ly Plant

[17] Murray, G. (1998), China: The Next Superpower, Chip Liv v: Londoi

[18] Murray, G. (2004), China's Population Control Po A Socio momic

Reassessment, PhD thesis, Liverpool John Moo es University: Liy crpool, UK.

[19] Phillipson, C. (1998), Reconstructing Old Age. ondon: Sag?

[20] Powell, J. (2001), Theorizing Gerontology: The

[21] Power and Social Policy in the United k tournal of Aging and Identity, Vol. 6, No. 3, pp.117-35.

[22] Powell, J.L. (2009) 'Social T/2 Aging, nd Health and Welfare Professionals: A Foucauldian "Toolkit", Jo nal of Applied Ćrontology, 28, 6, 669-682

[23] Powell, J. and Biggs, Vranagng Old Age: The Disciplinary Web of

[24] Power, Surveillap a Norman, Journal of Aging and Identity, Vol. 5, No. 1, pp.
3-13.

[25] Powell, J. nd Cook, I.G. ( 0 ), “A Tiger Behind and Coming Up Fast”: Govern ntalit $y d$ the Politics of Population Control in China, Journal of Aging and Identity, No. 2 sp. 79-90.

[26] Yor Bank (4), Averting the Old-Age Crisis, Oxford University Press: Oxford, UK.

[2, hen I (2004), China Faces Elderly Dilemma, China Daily, August 21. 